\title{
Estimating Enthalpy of Vaporization from Vapor Pressure using Trouton's Rule
}

\section{SUPPORTING INFORMATION}

\author{
Matthew MacLeod*, Martin Scheringer and Konrad Hungerbühler
}

Institute for Chemical and Bioengineering, ETH Zurich, CH-8093 Zurich, Switzerland

* - Corresponding Author. Phone: +41 446323171 FAX: +41 44632 1189 Email: macleod@chem.ethz.ch 


\section{Derivation of Equation 6 from Equations 3, 4 and 5 in the main text.}

Equation 3 in the main text is the Clausius-Clapeyron equation under the assumption that $\Delta \bar{H}_{\mathrm{VAP}}$ is constant over $\left[T^{\circ}, T_{\mathrm{B}}\right]$ :

$\ln P_{\mathrm{L}}^{\mathrm{o}}-\ln P_{\mathrm{L}}\left(T_{\mathrm{B}}\right)=\frac{\Delta \bar{H}_{\mathrm{VAP}}}{R}\left(\frac{1}{T_{\mathrm{B}}}-\frac{1}{T^{\mathrm{o}}}\right)$

Equation 4 is Trouton's rule:

$$
\Delta S_{\mathrm{VAP}}\left(T_{\mathrm{B}}\right)=\frac{\Delta H_{\mathrm{VAP}}\left(T_{\mathrm{B}}\right)}{T_{\mathrm{B}}} \cong 88 \mathrm{~J} \mathrm{~mol}^{-1} \mathrm{~K}^{-1}
$$

And, Equation 5 expresses the approximation that $\Delta \bar{H}_{\mathrm{VAP}}$ and $\Delta H_{\mathrm{VAP}}\left(T_{B}\right)$ are equal under the assumption that $\Delta \bar{H}_{\mathrm{VAP}}$ is constant over $\left[T^{\mathrm{o}}, T_{\mathrm{B}}\right]$ :

$$
\Delta \bar{H}_{\mathrm{VAP}} \approx \Delta H_{\mathrm{VAP}}\left(T_{\mathrm{B}}\right)
$$

Solving Equation S2 for $T_{\mathrm{B}}$ yields:

$$
T_{\mathrm{B}}=\frac{\Delta H_{\mathrm{VAP}}\left(T_{\mathrm{B}}\right)}{\Delta S_{\mathrm{VAP}}\left(T_{\mathrm{B}}\right)}
$$

Substituting Equation S4 into Equation S1 yields:

$$
\ln P_{\mathrm{L}}^{\mathrm{o}}-\ln P_{\mathrm{L}}\left(T_{\mathrm{B}}\right)=\frac{\Delta \bar{H}_{\mathrm{VAP}}}{R}\left(\frac{\Delta S_{\mathrm{VAP}}\left(T_{\mathrm{B}}\right)}{\Delta H_{\mathrm{VAP}}\left(T_{\mathrm{B}}\right)}-\frac{1}{T^{\mathrm{o}}}\right)
$$

Equation S5 can be rearranged to:

$$
\ln P_{\mathrm{L}}^{\mathrm{o}}-\ln P_{\mathrm{L}}\left(T_{\mathrm{B}}\right)=\frac{\Delta S_{\mathrm{VAP}}\left(T_{\mathrm{B}}\right)}{R} \frac{\Delta \bar{H}_{\mathrm{VAP}}}{\Delta H_{\mathrm{VAP}}\left(T_{\mathrm{B}}\right)}-\frac{\Delta \bar{H}_{\mathrm{VAP}}}{R T^{\circ}}
$$

Equation S3 implies that

$$
\frac{\Delta \bar{H}_{\mathrm{VAP}}}{\Delta H_{\mathrm{VAP}}\left(T_{\mathrm{B}}\right)} \approx 1
$$

Substituting Equation S7 into Equation S6 and solving for $\Delta \bar{H}_{\mathrm{VAP}}$ yields: 


$$
\Delta \bar{H}_{\mathrm{VAP}}^{\mathrm{TR}}=-R T^{\mathrm{o}} \ln P_{\mathrm{L}}^{\mathrm{o}}+R T^{\mathrm{o}} \ln P_{\mathrm{L}}\left(T_{\mathrm{B}}\right)+T^{\mathrm{o}} \Delta S_{\mathrm{VAP}}\left(T_{\mathrm{B}}\right)
$$

Where the superscript "TR" is used to indicate that $\Delta \bar{H}_{\mathrm{VAP}}$ is derived by using Trouton's rule. Equation S8 is identical to Equation 6 in the main text. 


\section{Derivation of Clausius-Clapeyron equation under the assumption of linear temperature dependence of $\Delta H_{\mathrm{VAP}}$ (Equations 9 and 10 in the main text).}

Up to Equation S13 this derivation follows the steps outlined by W.R. Salzman of the University of Arizona, (http://www.chem.arizona.edu/ salzmanr/480a/480ants/clapeyro/clapeyro.html).

Equation 7 in the main text expresses enthalpy of vaporization as a function of temperature $\left(\Delta H_{\mathrm{VAP}}(T)\right)$ assuming linear temperature dependence:

$$
\Delta H_{\mathrm{VAP}}^{\mathrm{LTD}}(T)=\Delta H_{\mathrm{VAP}}^{\mathrm{LTD}}\left(T_{1}\right)+\Delta C_{\mathrm{P}} \times\left(T-T_{1}\right)
$$

Substituting the right hand side of Equation S9 for $\Delta H_{\mathrm{VAP}}$ in Equation 2 yields the differential equation:

$\frac{d P_{\mathrm{L}}}{P_{\mathrm{L}}}=\frac{\Delta H_{\mathrm{VAP}}\left(T_{1}\right)+\Delta C_{\mathrm{P}} \times\left(T-T_{1}\right)}{R} \frac{d T}{T^{2}}$

Equation S10 can be expanded to:

$$
\frac{d P_{\mathrm{L}}}{P_{\mathrm{L}}}=\frac{\Delta H_{\mathrm{VAP}}\left(T_{1}\right)-T_{1} \Delta C_{\mathrm{P}}+T \Delta C_{\mathrm{P}}}{R} \frac{d T}{T^{2}}
$$

Gathering terms of the same order in terms of $T$ on the right hand side yields:

$\frac{d P_{\mathrm{L}}}{P_{\mathrm{L}}}=\frac{\Delta H_{\mathrm{VAP}}\left(T_{1}\right)-T_{1} \Delta C_{\mathrm{P}}}{R} \frac{d T}{T^{2}}+\frac{\Delta C_{\mathrm{P}}}{R} \frac{d T}{T}$

Equation S12 integrates to:

$\ln P_{\mathrm{L}}(T)-\ln P_{\mathrm{L}}\left(T_{1}\right)=\frac{\Delta H_{\mathrm{VAP}}\left(T_{1}\right)-T_{1} \Delta C_{\mathrm{P}}}{R}\left(\frac{1}{T_{1}}-\frac{1}{T}\right)+\frac{\Delta C_{\mathrm{P}}}{R} \ln \left(\frac{T}{T_{1}}\right)$

Setting $T=T^{\circ}$ and $T_{1}=T_{\mathrm{B}}$ in Equation $\mathrm{S} 13$ yields:

$\ln P_{\mathrm{L}}^{\mathrm{o}}-\ln P_{\mathrm{L}}\left(T_{\mathrm{B}}\right)=\frac{\Delta H_{\mathrm{VAP}}^{\mathrm{LTD}}\left(T_{\mathrm{B}}\right)-T_{\mathrm{B}} \Delta C_{\mathrm{P}}}{R}\left(\frac{1}{T_{\mathrm{B}}}-\frac{1}{T^{\mathrm{o}}}\right)+\frac{\Delta C_{\mathrm{P}}}{R} \ln \left(\frac{T^{\mathrm{o}}}{T_{\mathrm{B}}}\right)$

Equation S14 is Equation 9 in the main text. Equation S14 can be rearranged to solve for $\Delta H_{\mathrm{VAP}}^{L T D}\left(T_{\mathrm{B}}\right)$ : 


$$
\Delta H_{\mathrm{VAP}}^{\mathrm{LTD}}\left(T_{\mathrm{B}}\right)=\frac{T_{\mathrm{B}}\left(R T^{\mathrm{o}} \ln \left(\frac{P_{\mathrm{L}}\left(T_{\mathrm{B}}\right)}{P_{\mathrm{L}}^{\mathrm{o}}}\right)+\Delta C_{\mathrm{P}}\left(T_{\mathrm{B}}-T^{\mathrm{o}}\right)+\Delta C_{\mathrm{P}} T^{\mathrm{o}} \ln \left(\frac{T^{\mathrm{o}}}{T_{\mathrm{B}}}\right)\right)}{T_{\mathrm{B}}-T^{\mathrm{o}}}
$$

Setting $T=T_{\mathrm{B}}$ and $T_{1}=T^{\circ}$ in Equation S13 yields:

$\ln P_{\mathrm{L}}\left(T_{\mathrm{B}}\right)-\ln P_{\mathrm{L}}^{\mathrm{o}}=\frac{\Delta H_{\mathrm{VAP}}^{\mathrm{o}, \mathrm{LTD}}-T^{\mathrm{o}} \Delta C_{\mathrm{P}}}{R}\left(\frac{1}{T^{\mathrm{o}}}-\frac{1}{T_{\mathrm{B}}}\right)+\frac{\Delta C_{\mathrm{P}}}{R} \ln \left(\frac{T_{\mathrm{B}}}{T^{\mathrm{o}}}\right)$

Equation S16 is Equation 10 in the main text. Equation S16 can be rearranged to solve for $\Delta H_{\mathrm{VAP}}^{\mathrm{O}, \mathrm{LTD}}$ :

$$
\Delta H_{\mathrm{VAP}}^{\mathrm{o}, \mathrm{LTD}}=\frac{T^{\mathrm{o}}\left(R T_{\mathrm{B}} \ln \left(\frac{P_{\mathrm{L}}\left(T_{\mathrm{B}}\right)}{P_{\mathrm{L}}^{\mathrm{o}}}\right)+\Delta C_{\mathrm{P}}\left(T_{\mathrm{B}}-T^{\mathrm{o}}\right)-\Delta C_{\mathrm{P}} T_{\mathrm{B}} \ln \left(\frac{T_{\mathrm{B}}}{T^{\mathrm{o}}}\right)\right)}{T_{\mathrm{B}}-T^{\mathrm{o}}}
$$




\section{Derivation of Equation 13 from Equations 4, 8, 9 and 12.}

Equation 9 in the main text is:

$\ln P_{\mathrm{L}}^{\mathrm{o}}-\ln P_{\mathrm{L}}\left(T_{\mathrm{B}}\right)=\frac{\Delta H_{\mathrm{VAP}}^{\mathrm{LTD}}\left(T_{\mathrm{B}}\right)-T_{\mathrm{B}} \Delta C_{\mathrm{P}}}{R}\left(\frac{1}{T_{\mathrm{B}}}-\frac{1}{T^{\mathrm{o}}}\right)+\frac{\Delta C_{\mathrm{P}}}{R} \ln \left(\frac{T^{\mathrm{o}}}{T_{\mathrm{B}}}\right)$

Equation 12 is:

$\ln \left(\frac{T^{\mathrm{o}}}{T_{\mathrm{B}}}\right) \approx \alpha\left(1-\frac{T_{\mathrm{B}}}{T^{\mathrm{o}}}\right)$

Substituting Equation S19 in to Equation S18 and expanding yields:

$$
\ln P_{\mathrm{L}}^{\mathrm{o}}-\ln P_{\mathrm{L}}\left(T_{\mathrm{B}}\right)=\frac{\Delta H_{\mathrm{VAP}}^{\mathrm{LTD}}\left(T_{\mathrm{B}}\right)}{R}\left(\frac{1}{T_{\mathrm{B}}}-\frac{1}{T^{\mathrm{o}}}\right)-\frac{T_{\mathrm{B}} \Delta C_{\mathrm{P}}}{R}\left(\frac{1}{T_{\mathrm{B}}}-\frac{1}{T^{\mathrm{o}}}\right)+\frac{\alpha \Delta C_{\mathrm{P}}}{R}\left(1-\frac{T_{\mathrm{B}}}{T^{\mathrm{o}}}\right)
$$

Factor $T_{\mathrm{B}}$ out of the last term of Equation S20,

$\ln P_{\mathrm{L}}^{\mathrm{o}}-\ln P_{\mathrm{L}}\left(T_{\mathrm{B}}\right)=\frac{\Delta H_{\mathrm{VAP}}^{\mathrm{LTD}}\left(T_{\mathrm{B}}\right)}{R}\left(\frac{1}{T_{\mathrm{B}}}-\frac{1}{T^{\mathrm{o}}}\right)-\frac{T_{\mathrm{B}} \Delta C_{\mathrm{P}}}{R}\left(\frac{1}{T_{\mathrm{B}}}-\frac{1}{T^{\mathrm{o}}}\right)+\frac{\alpha T_{\mathrm{B}} \Delta C_{\mathrm{P}}}{R}\left(\frac{1}{T_{\mathrm{B}}}-\frac{1}{T^{\mathrm{o}}}\right) \ldots \mathrm{S} 21$

and collect terms:

$\ln P_{\mathrm{L}}^{\mathrm{o}}-\ln P_{\mathrm{L}}\left(T_{\mathrm{B}}\right)=\frac{\Delta H_{\mathrm{VAP}}^{\mathrm{LTD}}\left(T_{\mathrm{B}}\right)}{R}\left(\frac{1}{T_{\mathrm{B}}}-\frac{1}{T^{\mathrm{o}}}\right)+(\alpha-1) \frac{T_{\mathrm{B}} \Delta C_{\mathrm{P}}}{R}\left(\frac{1}{T_{\mathrm{B}}}-\frac{1}{T^{\mathrm{o}}}\right)$

Equation 4 in the main text is Trouton's rule which can be written as:

$\Delta H_{\mathrm{VAP}}\left(T_{\mathrm{B}}\right)=T_{\mathrm{B}} \Delta S_{\mathrm{VAP}}\left(T_{\mathrm{B}}\right)$

Equation 8 in the main text is:

$$
\Delta C_{\mathrm{P}}=\chi \times \Delta S_{\mathrm{VAP}}\left(T_{\mathrm{B}}\right)
$$

Substituting Equations S23 and S24 in Equation S22 yields:

$\ln P_{\mathrm{L}}^{\mathrm{o}}-\ln P_{\mathrm{L}}\left(T_{\mathrm{B}}\right)=\frac{T_{\mathrm{B}} \Delta S_{\mathrm{VAP}}\left(T_{\mathrm{B}}\right)}{R}\left(\frac{1}{T_{\mathrm{B}}}-\frac{1}{T^{\mathrm{o}}}\right)+(\alpha-1) \frac{\chi T_{\mathrm{B}} \Delta S_{\mathrm{VAP}}\left(T_{\mathrm{B}}\right)}{R}\left(\frac{1}{T_{\mathrm{B}}}-\frac{1}{T^{\mathrm{o}}}\right)$

Combine terms on the right hand side: 
$\ln P_{\mathrm{L}}^{\mathrm{o}}-\ln P_{\mathrm{L}}\left(T_{\mathrm{B}}\right)=(1-\chi+\alpha \chi) \frac{T_{\mathrm{B}} \Delta S_{\mathrm{VAP}}\left(T_{\mathrm{B}}\right)}{R}\left(\frac{1}{T_{\mathrm{B}}}-\frac{1}{T^{\mathrm{o}}}\right)$

Factor $1 / T_{\mathrm{B}}$ from the difference in the reciprocal temperatures:

$\ln P_{\mathrm{L}}^{\mathrm{o}}-\ln P_{\mathrm{L}}\left(T_{\mathrm{B}}\right)=(1-\chi+\alpha \chi) \frac{\Delta S_{\mathrm{VAP}}\left(T_{\mathrm{B}}\right)}{R}\left(1-\frac{T_{\mathrm{B}}}{T^{\mathrm{o}}}\right)$

and similarly factor out $1 / T^{\mathrm{o}}$ :

$\ln P_{\mathrm{L}}^{\mathrm{o}}-\ln P_{\mathrm{L}}\left(T_{\mathrm{B}}\right)=(1-\chi+\alpha \chi) \frac{\Delta S_{\mathrm{VAP}}\left(T_{\mathrm{B}}\right)}{R T^{\mathrm{o}}}\left(T^{\mathrm{o}}-T_{\mathrm{B}}\right)$

Rearrangement of Equation S28 yields:

$\frac{R T^{\mathrm{o}}\left(\ln P_{\mathrm{L}}^{\mathrm{o}}-\ln P_{\mathrm{L}}\left(T_{\mathrm{B}}\right)\right)}{(1-\chi+\alpha \chi)}=\Delta S_{\mathrm{VAP}}\left(T_{\mathrm{B}}\right)\left(T^{\mathrm{o}}-T_{\mathrm{B}}\right)$

Equation S29 is Equation 13 in the main text. 


\section{Derivation of Equation 14 from Equations 5, 6, 7, 8 and 13}

Equation 7 in the main text is:

$$
\Delta H_{\mathrm{VAP}}^{\mathrm{o}, \mathrm{LTD}}=\Delta H_{\mathrm{VAP}}^{\mathrm{LTD}}\left(T_{\mathrm{B}}\right)+\Delta C_{\mathrm{P}} \times\left(T^{\mathrm{o}}-T_{\mathrm{B}}\right)
$$

Equation 8 is:

$\Delta C_{\mathrm{P}}=\chi \times \Delta S_{\mathrm{VAP}}\left(T_{\mathrm{B}}\right)$

Substituting Equation S31 into Equation S30 yields:

$$
\Delta H_{\mathrm{VAP}}^{\mathrm{o}, \mathrm{LTD}}=\Delta H_{\mathrm{VAP}}^{\mathrm{LTD}}\left(T_{\mathrm{B}}\right)+\chi \Delta S_{\mathrm{VAP}}\left(T_{\mathrm{B}}\right)\left(T^{\mathrm{o}}-T_{\mathrm{B}}\right)
$$

Equation 13 from the main text is:

$$
\Delta S_{\mathrm{VAP}}\left(T_{\mathrm{B}}\right)\left(T^{\mathrm{o}}-T_{\mathrm{B}}\right)=\frac{R T^{\mathrm{o}}\left(\ln P_{\mathrm{L}}^{\mathrm{o}}-\ln P_{\mathrm{L}}\left(T_{\mathrm{B}}\right)\right)}{(1-\chi+\chi \alpha)}
$$

Substituting Equation S33 into Equation S32 yields:

$$
\Delta H_{\mathrm{VAP}}^{\mathrm{o}, \mathrm{LTD}}=\Delta H_{\mathrm{VAP}}^{\mathrm{LTD}}\left(T_{\mathrm{B}}\right)+\frac{\chi}{(1-\chi+\chi \alpha)} R T^{\mathrm{o}}\left(\ln P_{\mathrm{L}}^{\mathrm{o}}-\ln P_{\mathrm{L}}\left(T_{\mathrm{B}}\right)\right)
$$

Equation 6 in the main text is:

$$
\Delta \bar{H}_{\mathrm{VAP}}^{\mathrm{TR}}=-R T^{\mathrm{o}} \ln P_{\mathrm{L}}^{\mathrm{o}}+R T^{\mathrm{o}} \ln P_{\mathrm{L}}\left(T_{\mathrm{B}}\right)+T^{\mathrm{o}} \Delta S_{\mathrm{VAP}}\left(T_{\mathrm{B}}\right)
$$

Recognizing that $\Delta \bar{H}_{\mathrm{VAP}} \approx \Delta H_{\mathrm{VAP}}^{\mathrm{LTD}}\left(T_{\mathrm{B}}\right)$ (as illustrated in Figure 1 in the main text), we can substitute the right hand side of Equation S35 for $\Delta H_{\mathrm{VAP}}^{\mathrm{LTD}}\left(T_{\mathrm{B}}\right)$ in Equation S34 and collect terms, yielding:

$$
\Delta H_{\mathrm{VAP}}^{\mathrm{o}, \mathrm{LTD}}=(-1+\beta) R T^{\mathrm{o}} \ln P_{\mathrm{L}}^{\mathrm{o}}+(1-\beta) R T^{\mathrm{o}} \ln P_{\mathrm{L}}\left(T_{\mathrm{B}}\right)+T^{\mathrm{o}} \Delta S_{\mathrm{VAP}}\left(T_{\mathrm{B}}\right)
$$

Where:

$$
\beta=\frac{\chi}{(1-\chi+\alpha \chi)}
$$




\section{Empirical analysis of the relationship between $\Delta H_{\mathrm{VAP}}^{\mathrm{o}, \mathrm{LTD}}$ and $\ln \boldsymbol{P}_{\mathrm{L}}^{\circ}$}

To refine our theoretical understanding of the empirically derived Equation 1, we assembled a dataset of liquid or sub-cooled liquid vapor pressure at $298 \mathrm{~K}\left(P_{\mathrm{L}}^{\mathrm{o}}\right)$ and boiling point temperature $\left(T_{\mathrm{B}}\right)$ for 184 non-hydrogen bonding chemicals from data reported in handbooks $(1,2)$ and in literature $(3-6)$. For high boiling temperature substances in the dataset, such as polycyclic aromatic hydrocarbons and chlorinated dibenzodioxins and furans, we estimated $P_{\mathrm{L}}^{\mathrm{o}}$ from data for the solid substance using fugacity ratios calculated from heat capacity data (4). The complete dataset is given in Table S1. Chemical classes represented in the dataset include hydrocarbons, substituted benzenes, chlorinated aromatics, polycyclic aromatics, alcohols, ketones, ethers, and esters.

For all chemicals in the dataset we have estimated $\Delta \bar{H}_{\mathrm{VAP}}$ from Equation 3 and $\Delta H_{\mathrm{VAP}}^{\mathrm{LTD}}\left(T_{\mathrm{B}}\right)$ and $\Delta H_{\mathrm{VAP}}^{\mathrm{o} \text { LTD }}$ from the temperature dependent Clausius-Clapeyron equation (Equations 9 and 10 in the main text). We assume $\Delta C_{\mathrm{P}}=-70.4 \mathrm{~J} \mathrm{~mol}^{-1} \mathrm{~K}^{-1}$ for all substances as implied by Equations 4 and 8 when $\chi=-0.8$ (7). We selected this as the most appropriate value since it was derived from a set of chemicals with a wide range of volatility over the temperature range $\left[T^{\mathrm{o}}, T_{\mathrm{B}}\right]$. Linear regressions of $\Delta \bar{H}_{\mathrm{VAP}}, \Delta H_{\mathrm{VAP}}^{\mathrm{LTD}}\left(T_{\mathrm{B}}\right)$ and $\Delta H_{\mathrm{VAP}}^{\mathrm{o}, \mathrm{LTD}}$ against $\ln P_{\mathrm{L}}^{\mathrm{o}}$ from the dataset are summarized in Figure $\mathrm{S} 1$ and compared with $\Delta \bar{H}_{\mathrm{VAP}}^{\mathrm{TR}}$ from Equation 6 and $\Delta H_{\mathrm{VAP}}^{\mathrm{o}}$ from Equation 1. To ensure consistency with Trouton's rule the regressions were constrained to pass through $\Delta H_{\mathrm{VAP}}=26.2 \mathrm{~kJ} \mathrm{~mol}^{-1}$ $\left(=298 \mathrm{~K} \times 88 \mathrm{~J} \mathrm{~mol}^{-1} \mathrm{~K}^{-1}\right)$ for $P_{\mathrm{L}}^{\mathrm{o}}=101325 \mathrm{~Pa}$, since, for such a chemical, $T^{\mathrm{o}}=T_{\mathrm{B}}$ and $\Delta H_{\mathrm{VAP}}^{\mathrm{o}}=\Delta H_{\mathrm{VAP}}\left(T_{\mathrm{B}}\right)$. For clarity, only the regression lines are shown in Figure $\mathrm{S} 1$; separate plots showing the individual data points for the 184 chemicals are included below as Figures S2, S3 and S4. 


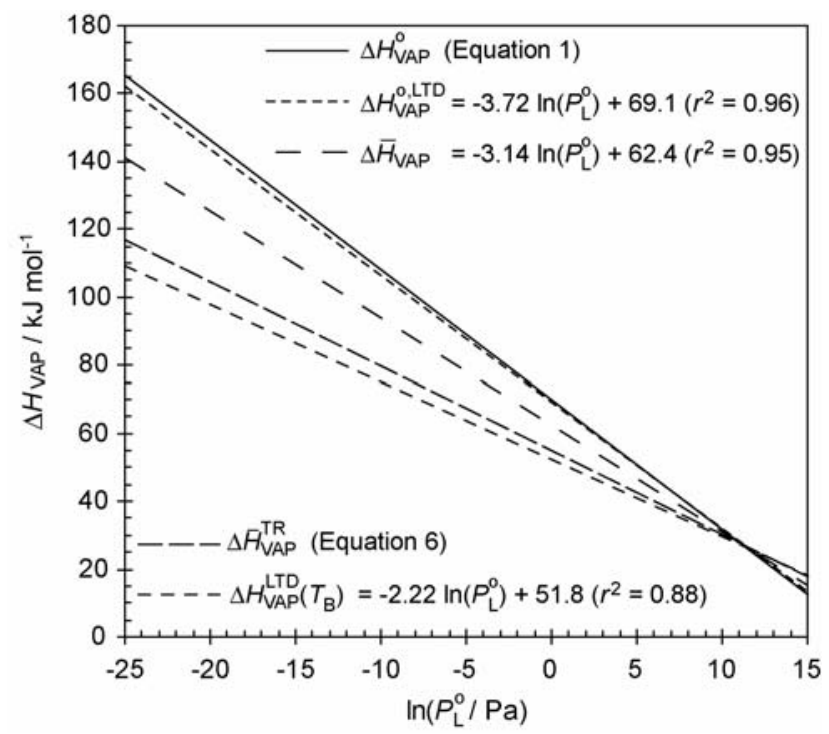

Figure S1. Relationships between estimates of enthalpy of vaporization ( $\left.\Delta H_{\mathrm{VAP}}\right)$ and the $\operatorname{logarithm}$ of liquid vapor pressure at $298 \mathrm{~K}\left(\ln P_{\mathrm{L}}^{\mathrm{o}}\right)$. Regression parameters for $\Delta \bar{H}_{\mathrm{VAP}}$, $\Delta H_{\mathrm{VAP}}^{\mathrm{o}, \mathrm{LTD}}$ and $\Delta H_{\mathrm{VAP}}^{\mathrm{LTD}}\left(T_{\mathrm{B}}\right)$ are estimated from the Clausius-Clapeyron equation (Equations 3, 9 and 10 respectively) and a dataset of 184 low polarity substances. (See Figures S2, S3 and S4 below.)

As shown in Figure $\mathrm{S} 1$, the empirical relationship between $\Delta H_{\mathrm{VAP}}^{\mathrm{o}, \mathrm{LTD}}$ and $\ln P_{\mathrm{L}}^{\mathrm{o}}$ is almost identical to the empirically-derived Equation 1. Therefore, from our dataset of $P_{\mathrm{L}}^{\mathrm{o}}$ and $T_{\mathrm{B}}$, the temperature dependent Clausius-Clapeyron equation (Equation 10), and established values of $\chi$ and $\Delta S_{\mathrm{VAP}}\left(T_{\mathrm{B}}\right)$ we independently arrived at the same result as Goss and Schwarzenbach (8). Agreement between these two empirical equations for $\Delta H_{\mathrm{VAP}}^{\mathrm{o}}$ demonstrates that the Goss and Schwarzenbach relationship (Equation 1) is consistent with earlier empirical relationships that independently established the values of $\chi(7)$ and $\Delta S_{\mathrm{VAP}}\left(T_{\mathrm{B}}\right)(9)$, and the Clausius Clapeyron equation under the assumption of linear temperature dependence of $\Delta H_{\mathrm{VAP}}$. 
Furthermore, Figure $\mathrm{S} 1$ demonstrates that $\Delta \bar{H}_{\mathrm{VAP}}^{\mathrm{TR}}$ lies close to $\Delta H_{\mathrm{VAP}}^{\mathrm{LTD}}\left(T_{\mathrm{B}}\right)$ over a wide range of $P_{\mathrm{L}}^{\circ}$. This supports the use of the approximation that $\Delta \bar{H}_{\mathrm{VAP}} \approx \Delta H_{\mathrm{VAP}}\left(T_{\mathrm{B}}\right)$, which is Equation 5 in the main text. 
Table S1. Physico-chemical property data for 184 substances used to estimate enthalpy of vaporization using the temperature dependent Clausius-Clapeyron equation.

\begin{tabular}{|c|c|c|c|c|c|c|}
\hline Ref. & Chemical Name & CAS \# & $T_{\mathbf{B}} / \mathbf{K}$ & $\begin{array}{c}\text { Fugacity } \\
\text { Ratio }\end{array}$ & $P_{\mathrm{L}}^{\mathrm{o}} / \mathbf{P a}$ & $\begin{array}{c}\ln \left(P_{\mathrm{L}}^{\circ} /\right. \\
\mathbf{P a})\end{array}$ \\
\hline $\mathrm{a}$ & benzene & $71-43-2$ & 353 & 1 & 12700 & 9.45 \\
\hline $\mathrm{a}$ & m-xylene & $108-38-3$ & 412 & 1 & 1100 & 7.00 \\
\hline $\mathrm{a}$ & p-xylene & $106-42-3$ & 411 & 1 & 1170 & 7.06 \\
\hline $\mathrm{a}$ & 1,2,3-trimethylbenzene & $526-73-8$ & 449 & 1 & 200 & 5.30 \\
\hline $\mathrm{a}$ & 1,2.4-trimethylbenzene & $95-63-6$ & 442 & 1 & 270 & 5.60 \\
\hline $\mathrm{a}$ & 1,3,5-trimethylbenzene & $108-67-8$ & 437 & 1 & 325 & 5.78 \\
\hline $\mathrm{a}$ & n-propylbenzene & $103-65-1$ & 432 & 1 & 450 & 6.11 \\
\hline $\mathrm{a}$ & isopropylbenzene & $98-82-8$ & 427 & 1 & 610 & 6.41 \\
\hline $\mathrm{a}$ & 1-ethyl-2-methylbenzene & $611-14-3$ & 438 & 1 & 330 & 5.80 \\
\hline $\mathrm{a}$ & 1-ethyl-3-methylbenzene & $620-14-4$ & 434 & 1 & 391 & 5.97 \\
\hline $\mathrm{a}$ & 1-ethyl-4-methylbenzene & $622-96-8$ & 435 & 1 & 395 & 5.98 \\
\hline $\mathrm{a}$ & isopropyl-4-methylbenzene & $99-87-6$ & 450 & 1 & 204 & 5.32 \\
\hline $\mathrm{a}$ & n-butylbenzene & $104-51-8$ & 456 & 1 & 137 & 4.92 \\
\hline $\mathrm{a}$ & isobutylbenzene & $538-93-2$ & 443 & 1 & 250 & 5.52 \\
\hline $\mathrm{a}$ & sec-butylbenzene & $135-98-8$ & 446 & 1 & 240 & 5.48 \\
\hline $\mathrm{a}$ & tert-butylbenzene & $98-06-6$ & 442 & 1 & 286 & 5.66 \\
\hline $\mathrm{a}$ & 1,2,3.4-tetramethylbenzene & $48-23-3$ & 478 & 1 & 45 & 3.81 \\
\hline $\mathrm{a}$ & 1,2,3,5-tetramethylbenzene & $527-53-7$ & 471 & 1 & 62 & 4.13 \\
\hline $\mathrm{a}$ & 1,2.4,5-tetramethylbenzene & $95-93-2$ & 469 & 1 & 66 & 4.19 \\
\hline $\mathrm{a}$ & n-pentylbenzene & $538-68-1$ & 478 & 1 & 44 & 3.78 \\
\hline $\mathrm{a}$ & pentamethylbenzene & $700-12-9$ & 504 & 0.511 & 18.63 & 2.92 \\
\hline $\mathrm{a}$ & n-hexylbenzene & $1077-16-3$ & 499 & 1 & 13.61 & 2.61 \\
\hline $\mathrm{a}$ & chlorobenzene & $108-90-7$ & 405 & 1 & 1580 & 7.37 \\
\hline $\mathrm{a}$ & 1,2-dichlorobenzene & $95-50-1$ & 453 & 1 & 170 & 5.14 \\
\hline $\mathrm{a}$ & 1,3-dichlorobenzene & $541-73-1$ & 446 & 1 & 260 & 5.56 \\
\hline $\mathrm{a}$ & 1,2,3-trichlorobenzene & $87-61-6$ & 491 & 0.53 & 52.83 & 3.97 \\
\hline $\mathrm{a}$ & 1,2,4-trichlorobenzene & $120-82-1$ & 486 & 1 & 40 & 3.69 \\
\hline $\mathrm{a}$ & 1,3,5-trichlorobenzene & $108-70-3$ & 481 & 0.41 & 78.05 & 4.36 \\
\hline $\mathrm{a}$ & 1,2,3,4-tetrachlorobenzene & $634-66-2$ & 527 & 0.6 & 8.67 & 2.16 \\
\hline $\mathrm{a}$ & 1,2,3,5-tetrachlorobenzene & $634-90-2$ & 519 & 0.51 & 19.22 & 2.96 \\
\hline a & pentachlorobenzene & $608-93-5$ & 550 & 0.23 & 0.9565 & -0.04 \\
\hline $\mathrm{a}$ & biphenyl & $92-54-2$ & 528 & 0.352 & 3.69 & 1.31 \\
\hline $\mathrm{a}$ & 2-chlorobiphenyl & $2051-61-7$ & 547 & 0.817 & 2.5 & 0.92 \\
\hline $\mathrm{a}$ & 3-chlorobiphenyl & $2051-61-8$ & 557 & 1 & 1 & 0.00 \\
\hline $\mathrm{a}$ & 4-chlorobiphenyl & $2051-62-9$ & 564 & 0.301 & 0.9 & -0.11 \\
\hline $\mathrm{a}$ & 3,3'-dichlorobiphenyl & $2050-67-1$ & 595 & 913 & 0.03 & -3.51 \\
\hline $\mathrm{a}$ & Indan & $496-11-7$ & 451 & 1 & 197 & 5.28 \\
\hline $\mathrm{a}$ & naphthalene & $91-20-3$ & 491 & 0.283 & 36.81 & 3.61 \\
\hline $\mathrm{a}$ & 1-methylnaphthalene & $90-12-0$ & 517 & 1 & 8.84 & 2.18 \\
\hline $\mathrm{a}$ & 2-methylnaphthalene & $91-57-6$ & 515 & 0.804 & 11.2 & 2.42 \\
\hline $\mathrm{a}$ & 1,2-dimethylnaphthalene & $573-98-8$ & 539 & 1 & 0.87 & -0.14 \\
\hline $\mathrm{a}$ & 1,4-dimethylnaphthalene & $571-58-4$ & 535 & 1 & 2.27 & 0.82 \\
\hline $\mathrm{a}$ & 2,3-dimethylnaphthalene & $581-40-8$ & 542 & 0.162 & 6.17 & 1.82 \\
\hline $\mathrm{a}$ & 2,6-dimethylnaphthalene & $581-40-2$ & 535 & 1.15 & 9.27 & 2.23 \\
\hline $\mathrm{a}$ & 1-ethylnaphthalene & $1127-76-0$ & 531 & 1 & 2.51 & 0.92 \\
\hline $\mathrm{a}$ & 2-ethylnaphthalene & $939-27-5$ & 524 & 1 & 4 & 1.39 \\
\hline
\end{tabular}




\begin{tabular}{|c|c|c|c|c|c|}
\hline bibenzyl & $103-29-7$ & 558 & 0.538 & 0.754 & -0.28 \\
\hline acenaphthene & $83-32-9$ & 550 & 0.198 & 1.52 & 0.42 \\
\hline acenaphthylene & $208-96-8$ & 538 & 0.217 & 4.14 & 1.42 \\
\hline fluorene & $86-73-7$ & 568 & 0.126 & 0.715 & -0.34 \\
\hline phenanthrene & $85-01-8$ & 612 & 0.177 & 0.113 & -2.18 \\
\hline fluoranthene & $206-44-0$ & 648 & 0.141 & 0.00872 & -4.74 \\
\hline chrysene & $218-01-9$ & 721 & 0.0053 & 0.000107 & -9.14 \\
\hline fluoranthene & $206-44-0$ & 648 & 0.141 & 0.00872 & -4.74 \\
\hline triethanolamine & $102-71-6$ & 608 & 1 & 0.00359 & -5.63 \\
\hline dibutyl phthalate & $84-74-2$ & 613 & 1 & 0.01 & -4.61 \\
\hline 1-dodecanol & $112-53-8$ & 532 & 1 & 0.11307 & -2.18 \\
\hline n-hexadecane & $544-76-3$ & 490 & 1 & 0.2 & -1.61 \\
\hline glycerol triacetate & $102-76-1$ & 531 & 1 & 0.33 & -1.11 \\
\hline dimethyl phthalate & $131-11-3$ & 557 & 1 & 0.4 & -0.92 \\
\hline thiodiglycol & $111-48-8$ & 556 & 1 & 0.43 & -0.84 \\
\hline Texanol & $25265-77-4$ & 517 & 1 & 0.8 & -0.22 \\
\hline 1-decanol & $112-30-1$ & 506 & 1 & 1 & 0.00 \\
\hline diethylene glycol & $111-46-6$ & 518 & 1 & 1 & 0.00 \\
\hline diethylene glycol monobutyl ether & $112-34-5$ & 498 & 1 & 3 & 1.10 \\
\hline dipropylene glycol (isomer mixture) & $110-98-5$ & 506 & 1 & 4 & 1.39 \\
\hline hexamethylene diisocyanate & $822-06-0$ & 528 & 1 & 4 & 1.39 \\
\hline p-anisaldehyde & $123-11-5$ & 523 & 1 & 5 & 1.61 \\
\hline diethyl sulfate & $64-67-5$ & 481 & 1 & 7.12 & 1.96 \\
\hline 1,3-butanediol & $107-88-0$ & 480 & 1 & 10 & 2.30 \\
\hline ethylene glycol & $107-21-1$ & 470 & 1 & 10 & 2.30 \\
\hline 1-octanol & $111-87-5$ & 467 & 1 & 11 & 2.40 \\
\hline n-dodecane & $112-40-3$ & 489 & 1 & 15.4 & 2.73 \\
\hline diethylene glycol monoethyl ether & $111-90-0$ & 475 & 1 & 17 & 2.83 \\
\hline 1,2-propylene glycol & $57-55-6$ & 461 & 1 & 17.2 & 2.84 \\
\hline benzyl alcohol & $100-51-6$ & 478 & 1 & 20 & 3.00 \\
\hline m-cresol & $108-39-4$ & 475 & 1 & 20 & 3.00 \\
\hline Linalool & $78-70-6$ & 471 & 1 & 21 & 3.04 \\
\hline diethylene glycol monomethyl ether & $111-77-3$ & 466 & 1 & 24 & 3.18 \\
\hline o-cresol & $95-48-7$ & 464 & 1 & 33 & 3.50 \\
\hline n-methylformamide & $123-39-7$ & 473 & 1 & 33.8 & 3.52 \\
\hline diethyl malonate & $105-53-3$ & 473 & 1 & 35.9 & 3.58 \\
\hline tributylamine & $102-82-9$ & 489 & 1 & 38.7 & 3.66 \\
\hline o-chlorobenzaldehyde & $89-98-5$ & 485 & 1 & 40 & 3.69 \\
\hline isophorone & $78-59-1$ & 483 & 1 & 40 & 3.69 \\
\hline n-methylpyrrolidone & $872-50-4$ & 475 & 1 & 45.5 & 3.82 \\
\hline Tetralin & $119-64-2$ & 479 & 1 & 53 & 3.97 \\
\hline dipropylene glycol monomethyl ether & $34590-94-8$ & 461 & 1 & 73.3 & 4.29 \\
\hline furfuryl alcohol & $98-00-0$ & 444 & 1 & 81.3 & 4.40 \\
\hline dimethyl sulfoxide & $67-68-5$ & 462 & 1 & 81.33 & 4.40 \\
\hline tetrahydrofurfuryl alcohol & $97-99-4$ & 451 & 1 & 106.6 & 4.67 \\
\hline 1-hexanol & $111-27-3$ & 430 & 1 & 110 & 4.70 \\
\hline 2-butoxyethanol & $111-76-2$ & 443 & 1 & 120 & 4.79 \\
\hline n,n-dimethylacetamide & $127-19-5$ & 438 & 1 & 173 & 5.15 \\
\hline diisobutyl ketone & $108-83-8$ & 441 & 1 & 220 & 5.39 \\
\hline 1-pentanol & $71-41-0$ & 411 & 1 & 293.3 & 5.68 \\
\hline furfural & $98-01-1$ & 434 & 1 & 294.6 & 5.69 \\
\hline 2-ethoxyethyl acetate & $111-15-9$ & 429 & 1 & 310 & 5.74 \\
\hline
\end{tabular}




\begin{tabular}{|c|c|c|c|c|c|c|}
\hline b & diethylene glycol dimethyl ether & $111-96-6$ & 435 & 1 & 394 & 5.98 \\
\hline b & 2-methyl-1-butanol & $137-32-6$ & 401 & 1 & 420 & 6.04 \\
\hline b & n,n-dimethylformamide & $68-12-2$ & 426 & 1 & 492 & 6.20 \\
\hline $\mathrm{b}$ & methyl amyl ketone & $110-43-0$ & 424 & 1 & 510 & 6.23 \\
\hline $\mathrm{b}$ & cyclohexanone & $108-94-1$ & 428 & 1 & 580 & 6.36 \\
\hline $\mathrm{b}$ & allyl glycidyl ether & $106-92-3$ & 427 & 1 & 630 & 6.45 \\
\hline $\mathrm{b}$ & n-amyl acetate & $628-63-7$ & 422 & 1 & 650 & 6.48 \\
\hline $\mathrm{b}$ & ethyl chloroacetate & $105-39-5$ & 417 & 1 & 673 & 6.51 \\
\hline $\mathrm{b}$ & methyl isoamyl ketone & $110-12-3$ & 418 & 1 & 690 & 6.54 \\
\hline b & 2-ethoxyethanol & $110-80-5$ & 408 & 1 & 710 & 6.57 \\
\hline b & 4-methyl-2-pentanol & $108-11-2$ & 404 & 1 & 710 & 6.57 \\
\hline b & dibutyl ether & $142-96-1$ & 413 & 1 & 801 & 6.69 \\
\hline b & o-xylene & $95-47-6$ & 417 & 1 & 880 & 6.78 \\
\hline $\mathrm{b}$ & 1-cyanobutane & $110-59-8$ & 415 & 1 & 972 & 6.88 \\
\hline $\mathrm{b}$ & methyl isobutenyl ketone & $141-79-7$ & 403 & 1 & 1095 & 7.00 \\
\hline $\mathrm{b}$ & m-xylene & $108-38-3$ & 412 & 1 & 1110 & 7.01 \\
\hline b & p-xylene & $106-42-3$ & 411 & 1 & 1160 & 7.06 \\
\hline $\mathrm{b}$ & 2-methoxyethanol & $109-86-4$ & 397 & 1 & 1270 & 7.15 \\
\hline b & ethylbenzene & $100-41-4$ & 409 & 1 & 1280 & 7.15 \\
\hline b & 3-picoline & $108-99-6$ & 416 & 1 & 1300 & 7.17 \\
\hline b & 4-picoline & $108-89-4$ & 417 & 1 & 1300 & 7.17 \\
\hline $\mathrm{b}$ & isobutanol & $78-83-1$ & 381 & 1 & 1386.6 & 7.23 \\
\hline $\mathrm{b}$ & n-butyl acetate & $123-86-4$ & 399 & 1 & 1533 & 7.33 \\
\hline b & propylene glycol monomethyl ether & $107-98-2$ & 391 & 1 & 1670 & 7.42 \\
\hline b & acetic acid & 64-19-7 & 391 & 1 & 2093 & 7.65 \\
\hline $\mathrm{b}$ & 2-butanol & $78-92-2$ & 372 & 1 & 2440 & 7.80 \\
\hline $\mathrm{b}$ & 1,1,2,2-tetrachloroethylene & $127-18-4$ & 394 & 1 & 2466 & 7.81 \\
\hline $\mathrm{b}$ & methyl isobutyl ketone & $108-10-1$ & 390 & 1 & 2650 & 7.88 \\
\hline $\mathrm{b}$ & 1-propanol & $71-23-8$ & 370 & 1 & 2800 & 7.94 \\
\hline $\mathrm{b}$ & toluene & $108-88-3$ & 383 & 1 & 3780 & 8.24 \\
\hline $\mathrm{b}$ & methyl tert-butyl ketone & $75-97-8$ & 379 & 1 & 4270 & 8.36 \\
\hline b & methyl propyl ketone & $107-87-9$ & 375 & 1 & 4720 & 8.46 \\
\hline b & 1,4-dioxane & $123-91-1$ & 374 & 1 & 5080 & 8.53 \\
\hline $\mathrm{b}$ & formic acid & $64-18-6$ & 373 & 1 & 5680 & 8.64 \\
\hline $\mathrm{b}$ & n-heptane & $142-82-5$ & 371 & 1 & 6093 & 8.71 \\
\hline b & methyl isopropyl ketone & $563-80-4$ & 367 & 1 & 6960 & 8.85 \\
\hline b & dimethyl carbonate & $616-38-6$ & 363 & 1 & 7380 & 8.91 \\
\hline b & diacetyl & $431-03-8$ & 361 & 1 & 7450 & 8.92 \\
\hline $\mathrm{b}$ & triethylamine & $121-44-8$ & 362 & 1 & 7610 & 8.94 \\
\hline b & diethyl sulfide & $352-93-2$ & 365 & 1 & 7780 & 8.96 \\
\hline $\mathrm{b}$ & acetic acid, isopropyl ester & $108-21-4$ & 361 & 1 & 7890 & 8.97 \\
\hline $\mathrm{b}$ & 1,1,2-trichloroethylene & 79-01-6 & 359 & 1 & 9200 & 9.13 \\
\hline $\mathrm{b}$ & 1,2-dimethoxyethane & $110-71-4$ & 355 & 1 & 9270 & 9.13 \\
\hline $\mathrm{b}$ & 2,2,2-trifluoroethanol & $75-89-8$ & 378 & 1 & 10100 & 9.22 \\
\hline $\mathrm{b}$ & fluorobenzene & $462-06-6$ & 358 & 1 & 10200 & 9.23 \\
\hline $\mathrm{b}$ & diisopropylamine & $108-18-9$ & 356 & 1 & 10820 & 9.29 \\
\hline $\mathrm{b}$ & methyl propionate & $554-12-1$ & 352 & 1 & 11200 & 9.32 \\
\hline $\mathrm{b}$ & n-butylamine & $109-73-9$ & 350 & 1 & 12230 & 9.41 \\
\hline $\mathrm{b}$ & ethyl acetate & $141-78-6$ & 350 & 1 & 12490 & 9.43 \\
\hline b & benzene & $71-43-2$ & 353 & 1 & 12690 & 9.45 \\
\hline b & methyl ethyl ketone & $78-93-3$ & 352 & 1 & 12710 & 9.45 \\
\hline $\mathrm{b}$ & carbon tetrachloride & $56-23-5$ & 349 & 1 & 15333 & 9.64 \\
\hline
\end{tabular}




\begin{tabular}{|c|c|c|c|c|c|c|}
\hline$b$ & 1,1,1-trichloroethane & $71-55-6$ & 347 & 1 & 16530 & 9.71 \\
\hline $\mathrm{b}$ & methanol & $67-56-1$ & 337 & 1 & 16933 & 9.74 \\
\hline $\mathrm{b}$ & n-hexane & $110-54-3$ & 341 & 1 & 20170 & 9.91 \\
\hline $\mathrm{b}$ & tetrahydrofuran & $109-99-9$ & 339 & 1 & 21600 & 9.98 \\
\hline $\mathrm{b}$ & acetone & $67-64-1$ & 329 & 1 & 30800 & 10.34 \\
\hline $\mathrm{b}$ & 1,1,2-trichlorotrifluoroethane & 76-13-1 & 320 & 1 & 48266 & 10.78 \\
\hline $\mathrm{b}$ & dichloromethane & $75-09-2$ & 312 & 1 & 58000 & 10.97 \\
\hline $\mathrm{b}$ & propylene oxide & $75-56-9$ & 307 & 1 & 58920 & 10.98 \\
\hline $\mathrm{b}$ & n-pentane & $109-66-0$ & 309 & 1 & 68330 & 11.13 \\
\hline $\mathrm{b}$ & diethyl ether & $60-29-7$ & 307 & 1 & 71600 & 11.18 \\
\hline $\mathrm{b}$ & furan & $110-00-9$ & 304 & 1 & 80000 & 11.29 \\
\hline $\mathrm{b}$ & tetramethylsilane & $75-76-3$ & 300 & 1 & 95600 & 11.47 \\
\hline $\mathrm{c}$ & anthracene & $120-12-7$ & 613 & 0.015 & 0.05188 & -2.96 \\
\hline c & pyrene & $129-00-0$ & 633 & 0.14 & 0.00415 & -5.48 \\
\hline $\mathrm{c}$ & triphenylene & $217-59-4$ & 711 & 0.061 & $6.82 \mathrm{E}-05$ & -9.59 \\
\hline $\mathrm{c}$ & benz[a]anthracene & $56-55-3$ & 708 & 0.12 & 0.000225 & -8.40 \\
\hline c & chrysene & $218-01-9$ & 721 & 0.028 & 4.24E-05 & -10.07 \\
\hline $\mathrm{c}$ & benzo[a]pyrene & $50-32-8$ & 768 & 0.21 & $3.46 \mathrm{E}-06$ & -12.57 \\
\hline $\mathrm{c}$ & Dibenz[a,h]anthracene & $53-70-1$ & 797 & 0.026 & $7.43 \mathrm{E}-08$ & -16.42 \\
\hline $\mathrm{d}$ & dibenzo-p-dioxin & $262-23-4$ & 581 & 0.14 & 0.392857 & -0.93 \\
\hline $\mathrm{d}$ & 1-chlorodibenzo-p-dioxin & $39227-53-7$ & 613 & 0.222 & 0.054054 & -2.92 \\
\hline $\mathrm{d}$ & 2-chlorodibenzo-p-dioxin & $39227-54-8$ & 614 & 0.235 & 0.07234 & -2.63 \\
\hline $\mathrm{d}$ & 2,3-dichlorodibenzo-p-dioxin & $29446-15-9$ & 656 & 0.0513 & 0.007602 & -4.88 \\
\hline $\mathrm{d}$ & 2,7-dichlorodibenzo-p-dioxin & $33857-26-0$ & 672 & 0.0291 & 0.004124 & -5.49 \\
\hline $\mathrm{d}$ & 2,8-dichlorodibenzo-p-dioxin & $38964-22-6$ & 680 & 0.0614 & 0.00228 & -6.08 \\
\hline $\mathrm{d}$ & 1,3,7-trichlorodibenzo-p-dioxin & $67028-17-5$ & 696 & 0.0402 & 0.000896 & -7.02 \\
\hline $\mathrm{d}$ & 1,2,4-trichlorodibenzo-p-dioxin & $39227-58-2$ & 673 & 0.1465 & 0.000683 & -7.29 \\
\hline $\mathrm{d}$ & 1,2,3,4-tetrachlorodibenzo-p-dioxin & $30756-58-8$ & 717 & 0.019 & 0.000337 & -8.00 \\
\hline $\mathrm{d}$ & $2,3,7,8$-tetrachlorodibenzo-p-dioxin & $1746-01-6$ & 744 & 0.00312 & $6.41 \mathrm{E}-05$ & -9.66 \\
\hline $\mathrm{d}$ & octachlorodibenzo-p-dioxin & $3268-87-9$ & 808 & $7.28 \mathrm{E}-5$ & $1.51 \mathrm{E}-06$ & -13.40 \\
\hline $\mathrm{d}$ & dibenzofuran & $132-64-9$ & 546 & 0.304 & 1.151316 & 0.14 \\
\hline $\mathrm{d}$ & 3,6-dichlorodibenzofuran & $74978-40-4$ & 655 & 0.0177 & 0.011299 & -4.48 \\
\hline $\mathrm{d}$ & 2,4,8-trichlrorodibenzofuran & $54589-71-8$ & 690 & 0.0311 & 0.002926 & -5.83 \\
\hline $\mathrm{d}$ & octachlorodibenzofuran & $39001-02-0$ & 835 & 0.00057 & $8.74 \mathrm{E}-07$ & -13.95 \\
\hline \multicolumn{7}{|c|}{$\mathrm{a}-$ Reference $(2)$} \\
\hline \multicolumn{7}{|c|}{$\mathrm{b}$ - Reference $(1)$} \\
\hline \multicolumn{7}{|c|}{$\mathrm{c}-T_{\mathrm{B}}$ from reference $(2), P_{\mathrm{L}}^{\mathrm{o}}$ and fugacity ratio from reference (5) } \\
\hline \multicolumn{7}{|c|}{$\mathrm{d}-T_{\mathrm{B}}$ from reference $(6), P_{\mathrm{L}}^{\mathrm{o}}$ calculated from $P_{\mathrm{S}}^{\mathrm{o}}$ reported in reference $(3)$ using fugacity } \\
\hline
\end{tabular}




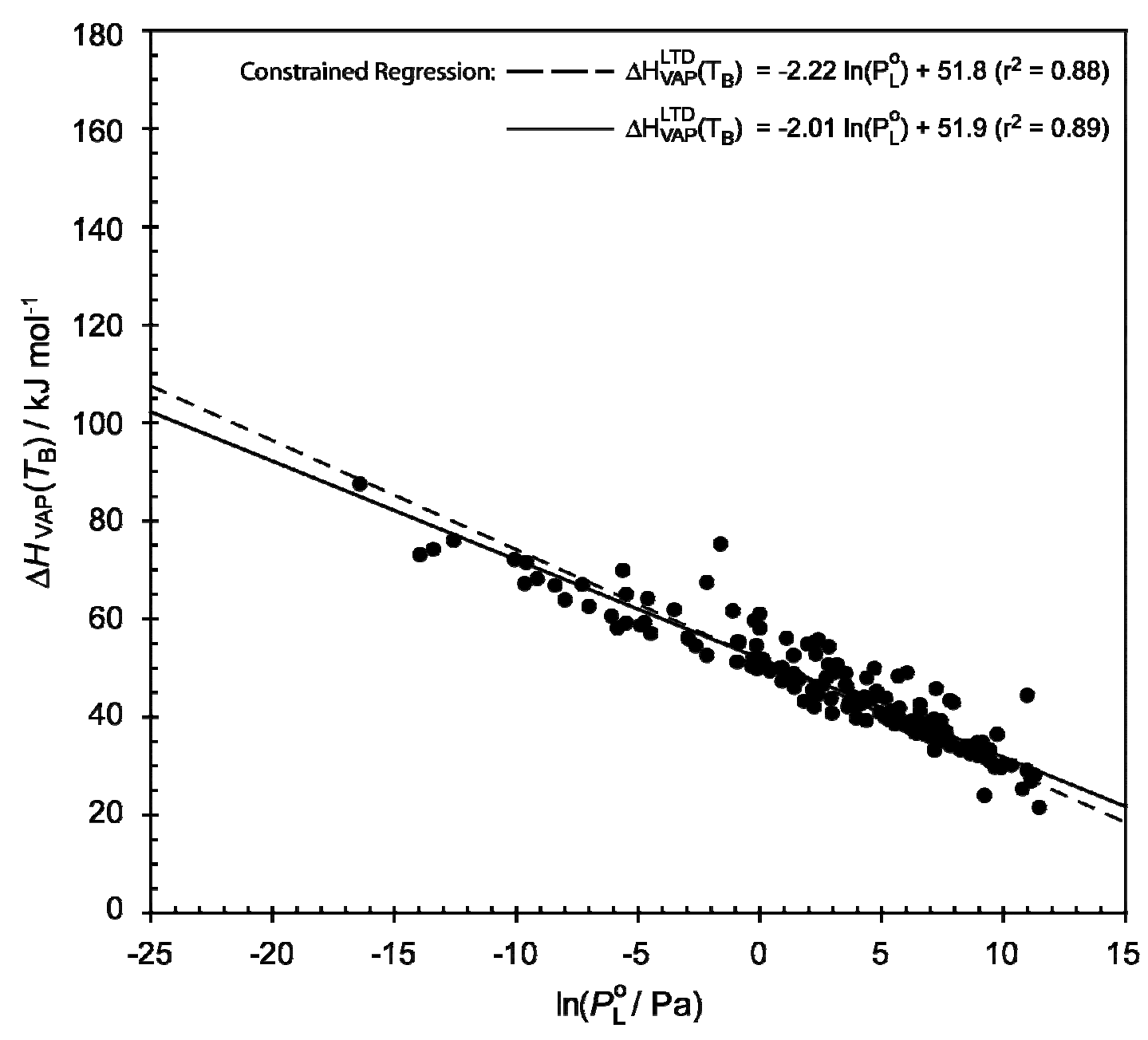

Figure S2. Enthalpy of vaporization at the normal boiling temperature estimated using the temperature dependent Clausius-Clapeyron equation (Equation S15, which is a form of Equation 9 in the main text) versus the logarithm of vapor pressure for the 184 substances listed in Table S1. Regression equations are shown for normal linear regression and regression constrained to pass through $\Delta H_{\mathrm{VAP}}=\left(298 \mathrm{~K} \times 88 \mathrm{~J} \mathrm{~mol}^{-1} \mathrm{~K}^{-1}\right)$ for $P_{\mathrm{L}}^{\mathrm{o}}=101325 \mathrm{~Pa}$, as dictated by Trouton's Rule. 


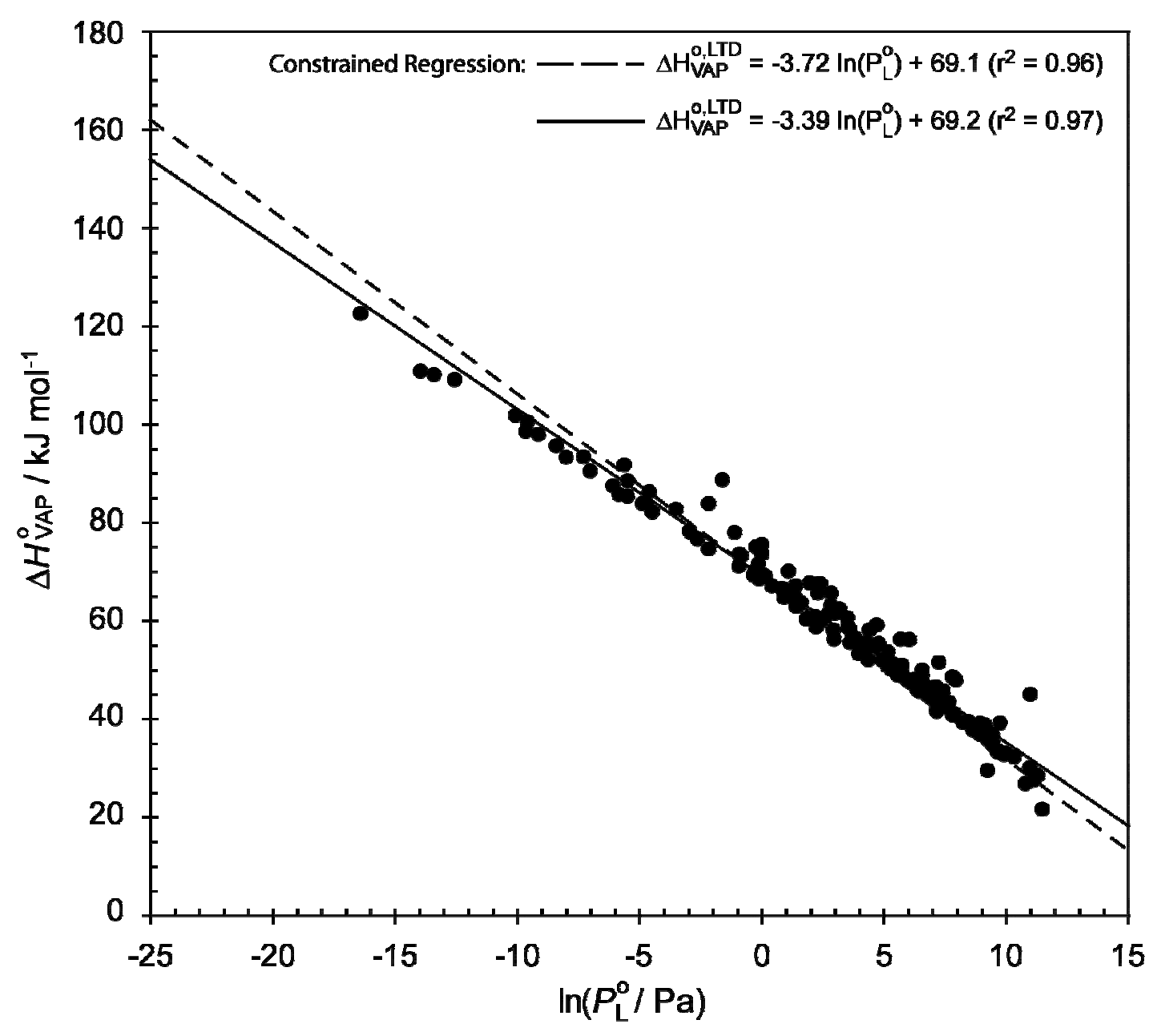

Figure S3. Enthalpy of vaporization at $298 \mathrm{~K}$ estimated using the temperature dependent Clausius-Clapeyron equation (Equation S17, which is a form of Equation 10 in the main text) versus the logarithm of vapor pressure for the 184 substances listed in Table S1. Regression equations are shown for normal linear regression and regression constrained to pass through $\Delta H_{\mathrm{VAP}}=\left(298 \mathrm{~K} \times 88 \mathrm{~J} \mathrm{~mol}^{-1} \mathrm{~K}^{-1}\right)$ for $P_{\mathrm{L}}^{\mathrm{o}}=101325 \mathrm{~Pa}$, as dictated by Trouton's Rule. 


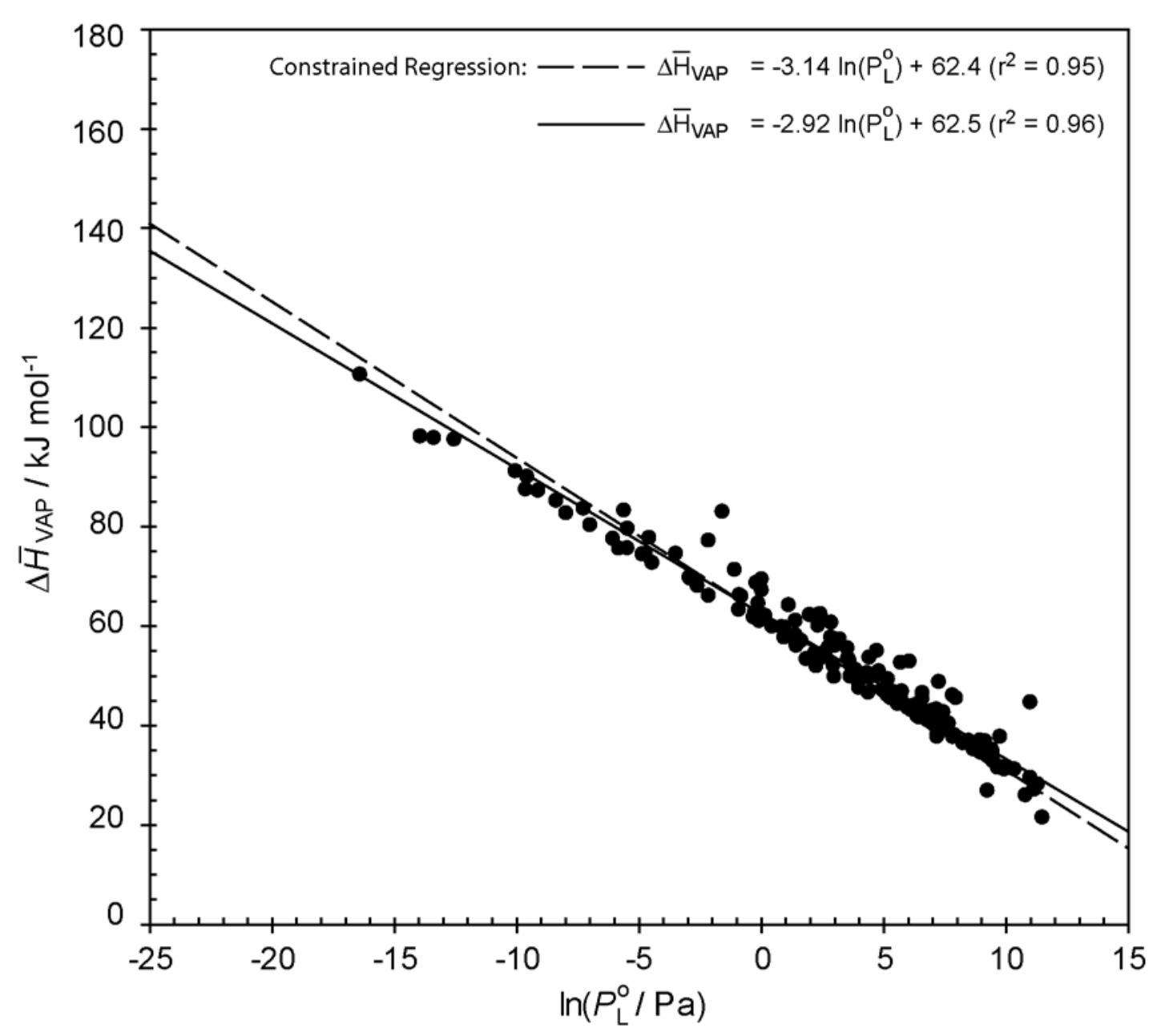

Figure S4. Enthalpy of vaporization estimated using the Clausius-Clapeyron equation assuming $\Delta H_{\mathrm{VAP}}$ is constant over the range $\left[T^{\circ}, T_{\mathrm{B}}\right]$ (Equation 3 in the main text) versus the logarithm of vapor pressure for the 184 substances listed in Table S1. Regression equations are shown for normal linear regression and regression constrained to pass through $\Delta H_{\mathrm{VAP}}=\left(298 \mathrm{~K} \times 88 \mathrm{~J} \mathrm{~mol}^{-1} \mathrm{~K}^{-1}\right)$ for $P_{\mathrm{L}}^{\mathrm{o}}=101325 \mathrm{~Pa}$, as dictated by Trouton's Rule. 


\section{Approximation of the logarithm function by the first term of the Taylor series}

expansion

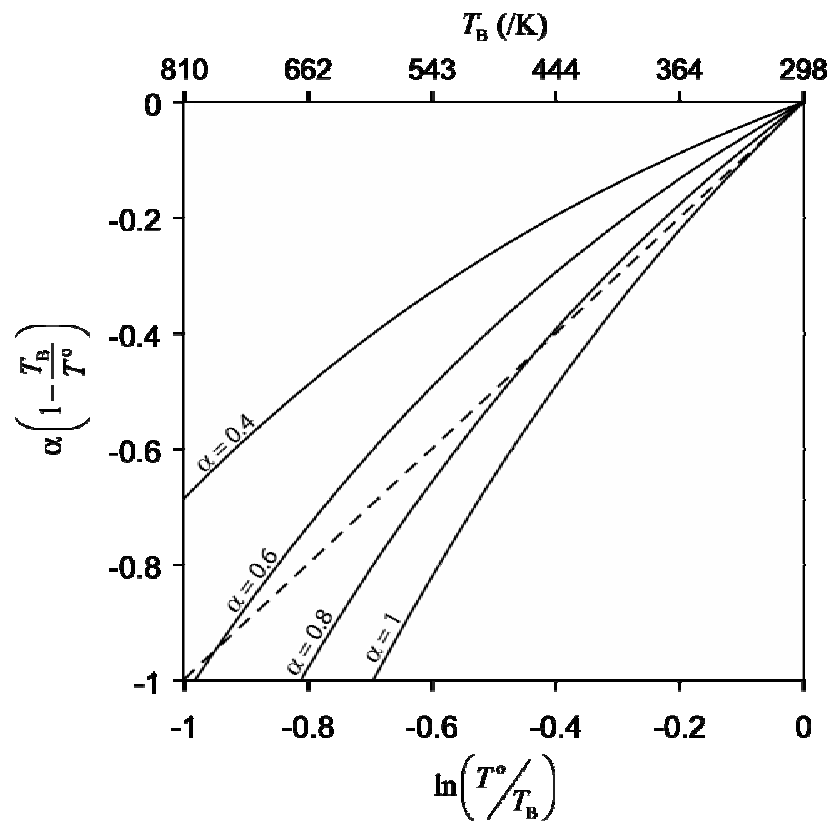

Figure S5: Approximation of $\ln \left(T^{\mathrm{o}} / T_{\mathrm{B}}\right)$ over a range of boiling point temperatures between $298 \mathrm{~K}$ and $810 \mathrm{~K}$, the approximate range of boiling point temperatures in the 184 substance dataset in Table S1. $\alpha=0.6$ provides a good estimation of $\ln \left(T^{\mathrm{o}} / T_{\mathrm{B}}\right)$ over this range of $T_{\mathrm{B}}$. 


\section{Evaluation of the accuracy of Equation 13}

Equation 13 in the main text can be rearranged to estimate $\ln P_{\mathrm{L}}^{\mathrm{o}}$ from $T_{\mathrm{B}}$ :

$\ln P_{\mathrm{L}}^{\mathrm{o}}=\frac{(1-\chi+\alpha \chi) \Delta S_{\mathrm{VAP}}\left(T_{\mathrm{B}}\right)\left(T^{\mathrm{o}}-T_{\mathrm{B}}\right)}{R T^{\mathrm{o}}}+\ln P_{\mathrm{L}}\left(T_{\mathrm{B}}\right)$.

Figure S6 compares $\ln P_{\mathrm{L}}^{\mathrm{o}}$ calculated with Equation S38 against measured data for the 184 substances in Table $\mathrm{S} 1$ using established values of $\Delta S_{\mathrm{VAP}}\left(T_{\mathrm{B}}\right)=88 \mathrm{~J} \mathrm{~mol}^{-1} \mathrm{~K}^{-1}$, $\chi=-0.75$ and $\alpha=0.6$.

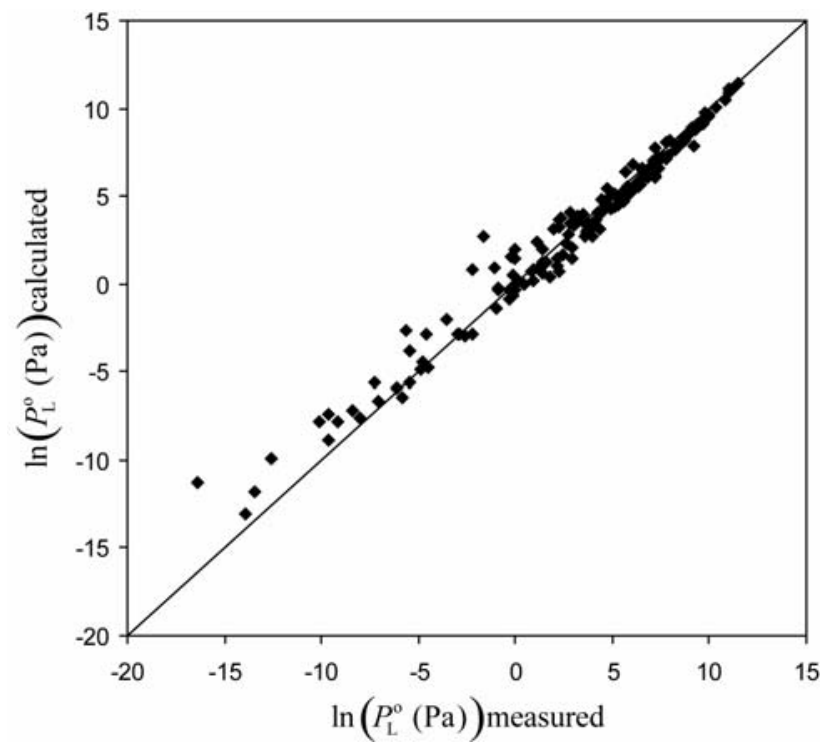

Figure S6: Comparison of measured $\ln P_{\mathrm{L}}^{\mathrm{o}}$ and that calculated with Equation S38, which is a form of Equation 13 in the main text. 


\section{References}

(1) Knovel Library Knovel Sample Book Knovel Corporation: Norwich, NY, 2003; http://knovel.com/knovel2/Toc.jsp?BookID=901.

(2) Mackay, D.; Shiu, W.-Y.; Ma, K.-C. Physical-Chemical Properties and Environmental Fate Handbook Chapman \& Hall/CRCnetBASE: Boca Raton, FL, 2000.

(3) Rordorf, B. F., Prediction of vapor pressures, boiling points and enthalpies of fusion for twenty-nine halogenated dibenxo-p-dioxins and fifty-five dibenzofurans by a vapor pressure correlation method. Chemosphere 1989 18, 783 - 788.

(4) Van Noort, P. C. M., Fugacity ratio estimations for high-melting rigid aromatic compounds. Chemosphere 2004 56, 7-12.

(5) Allen, J. O.; Sarofim, A. F.; Smith, K. A., Thermodynamic properties of polycyclic aromatic hydrocarbons in the subcooled liquid state. Polycyclic Aromatic Compounds 1999 13, 261-283.

(6) Iorish, V. S.; Dorofeeva, O. V.; Moiseeva, N. F., Thermodynamic properties of dibenzo-p-dioxin, dibenzofurans, and their polychlorinated derivatives in the gaseous and condensed phases. 2. Thermodynamic properties of condensed compounds. Journal of Chemical and Engineering Data 2001 46, 286-298.

(7) Mackay, D.; Bobra, A.; Chan, D. W.; Shiu, W. Y., Vapor pressure correlations for low-volatility environmental chemicals. Environ. Sci. Technol. 1982 16, 645-649.

(8) Goss, K. U.; Schwarzenbach, R. P., Empirical prediction of heats of vaporization and heats of adsorption of organic compounds. Environ. Sci. Technol. 1999 33, 3390-3393.

(9) Nash, L. K., Trouton and T-H-E Rule. Journal of Chemical Education $198461,981-984$. 